\title{
Clinical and ultrasound findings in patients with calcium pyrophosphate dihydrate deposition disease
}

\author{
Paulina Vele ${ }^{1}$, Siao-Pin Simon ${ }^{1,2}$, Laura Damian², Ioana Felea², Laura Muntean ${ }^{1,2}$, \\ Ileana Filipescu ${ }^{1,2}$, Simona Rednic ${ }^{1,2}$
}

1"Iuliu Haţieganu" University of Medicine and Pharmacy, ${ }^{2}$ Emergency County Hospital, Rheumatology Department, Cluj-Napoca, Romania

\begin{abstract}
Aim: To evaluate the presence and distribution of calcium pyrophosphate (CPP) deposits in joints commonly affected by CPP deposition (CPPD) disease (acromio-clavicular, gleno-humeral, wrists, hips, knees, ankles, and symphysis pubis joints) using ultrasound (US). Material and methods: Thirty consecutive patients fulfilling McCarty diagnostic criteria for CPPD were consecutively enrolled in the study. The data registered using the US included the affected joints, the calcification site, and the pattern of calcification (thin hyperechoic bands, parallel to the surface of the hyaline cartilage, hyperechoic spots, and hyperechoic nodular or oval deposits). The presence of CPP crystals in knees was confirmed by polarized light microscopy examination of the synovial fluid and radiographs of the knees were performed in all patients. Results: In 30 patients, 390 joints were scanned, (13 joints in every patient). The mean \pm standard deviation number of joints with US CPPD evidence per patient was $2.93 \pm 1.8$ (range 1-9). The knee was the most common joint involved both clinically and using US examination. The second US pattern (with hyperechoic spots) was the most frequent. Fibrocartilage calcifications were more common than hyaline calcification. Using radiography as reference method, the sensitivity and specificity of US for diagnosis CPPD in knees was $79.31 \%, 95 \mathrm{CI}(66.65 \%-88.83 \%)$, and $14.29 \%, 95 \mathrm{CI}(1.78 \%-42.81 \%)$, respectively. Conclusions: The knee is the most frequent joint affected by CPPD. The second ultrasound pattern is the most common. CPPD affects the fibrocartilage to a greater extent than the hyaline cartilage.
\end{abstract}

Keywords: calcium pyrophosphate dihydrate; ultrasonography; radiography

\section{Introduction}

Calcium pyrophosphate deposition (CPPD) disease is characterized by deposition of calcium pyrophosphate crystals inside the hyaline cartilage, fibrocartilage, and soft tissues [1,2]. The most common sites for CPPD are the meniscal fibrocartilage of the knee, hyaline cartilage of the femoral condyles, triangular fibrocartilage of the wrist, symphysis pubis discus, acromio-clavicular fibrocartilage, and the hyaline cartilage of the shoulder, hip, and ankle $[3,4]$.

Received 27.08.2017 Accepted 03.12.2017

Med Ultrason

2018, Vol. 20, No 2, 159-163

Corresponding author: Paulina Vele

Rheumatology Department, 2-4 Clinicilor Street

400006, Cluj-Napoca, Romania

Phone: +40746-093655

E-mail: paulina.vele@yahoo.com
The standard tool for diagnosis is synovial fluid analysis with identification of calcium pyrophosphate (CPP) crystals by microscopy using compensated polarized light [5]. McCarty criteria used for a definite diagnosis of CPPD require the presence of CPP crystals in the synovial fluid analysis and typical radiographic calcifications [1]. The presence of a thin, parallel line with the articular surface (hyaline cartilage calcification) and/or punctate fibrocartilage calcification in knees, symphysis pubis, wrists, hips, elbows, and ankles is considered as evidence of CPPD $[4,6]$.

Ultrasound (US) evaluation of CPPD is of great interest because of the high sensitivity and specificity of the method compared to conventional radiography. US was included in EULAR recommendations as an alternative imaging technique in the CPPD diagnostic approach $[2,7,8]$. Three US patterns of CPP deposits are described: a thin hyperechoic band, parallel to the surface on the 
inside of the hyaline cartilage; hyperechoic spots in the fibrocartilage; and hyperechoic nodular or oval deposits in bursae and articular recesses [7,9].

Due to the absence of histopathological correlations, no agreement in US evaluation in CPPD exists. It is not clear which joints should be screened, which definition should be used, and if the US changes are specific for CPPD.

The aim of this study was to systematically examine by US all the joints potentially affected by CPPD in patients with CPPD following the McCarty criteria, in order to assess the presence and distribution of the CPP deposits. Our secondary aim was to correlate the clinical data with US findings.

\section{Material and methods}

This prospective study was performed in the Rheumatology Department, Emergency Clinical County Hospital Cluj-Napoca, Romania, between January 2015 and January 2017. Patients who fulfilled McCarty diagnostic criteria for CPPD were consecutively enrolled in the study. Exclusion criteria were patients under 18 years of age, the presence of gout or/and other inflammatory rheumatic diseases.

\section{Clinical examination}

All patients were evaluated by the same rheumatologist. Data about age (years), sex, weight (kg), height $\left(\mathrm{m}^{2}\right)$, body mass index (BMI), type of joint involvement (monoarticular - 1 joint, oligoarticular - 2-4 joints, polyarticular $\geq 5$ joints), the form of arthritis (acute, chronic), and symptoms duration were recorded.

\section{US examination}

US examinations were performed using an Esaote My Lab25 Gold with 5-10 and 10-18 MHz linear probes by the same examiner. The US scanning technique was performed according to the standard musculoskeletal ultrasound guidelines $[10,11]$. In every patient 13 joints were systematically examined: the acromio-clavicular joints (longitudinal scans), the gleno-humeral joints (posterior transverse scan for posterior glenoid labrum), wrists for the triangular fibrocartilage (longitudinal scan with the wrist in pronation and slight radial deviation, on the lateral aspect of the wrist), the hips (anterior longitudinal and transverse scan with the hip in a neutral position for hyaline cartilage and fibrocartilage), the knees (anterior scans with the knee in maximum flexion for the hyaline cartilage and medial and lateral scans with the knee flexed at $30^{\circ}$ ), the ankles (anterior and transverse scans for the hyaline cartilage) and the symphysis pubis (transverse scan for the fibrocartilage).

The protocol included searching for calcific deposits on cartilage and fibrocartilage. Frediani's US patterns [7] were used for detecting evidence of CPPD: pattern 1 - thin hyperechoic bands, parallel to the surface of the hyaline cartilage; pattern 2 - thin hyperechoic spots in fibrocartilage; and pattern 3 - homogeneous hyperechoic nodular or oval deposits in the fibrocartilage. (fig 1). The US registered data included the affected joint, the pattern of calcification $(1,2$, or 3$)$ and the calcification site (fibrocartilage, hyaline cartilage).

\section{Radiographic examination}

A radiologist experienced in evaluating musculoskeletal system disorders studied the radiographic images. Hyaline cartilage calcification and punctate fibrocartilage calcification in at least one of the joints were considered proof of CPPD. Hyaline cartilage calcification was defined as a radiodense linear, parallel with the subchondral bone, in the middle zone of the cartilage, while fibrocartilage calcification is described as more dense, punctuate $[6,12]$.

The synovial fluid analysis was performed using polarized light microscopy. Crystals with a parallelepipedic or rhomboid shape and weak birefringence were considered as CPP crystals.

All patients included in the study gave their consent for participation, and the University Ethics Committee approved this research.

Statistical analysis was performed using Microsoft Excel. Results are presented as a mean \pm standard deviation (SD). We considered a level of $\mathrm{p}<0.05$ statistically significant.

\section{Results}

Thirty patients with CPPD were included in the study. The demographics and clinical data of the enrolled patients are detailed in Table I. All patients had knee effusion that was available for aspiration and for synovial fluid analysis.

Table I. General characteristics of study participants

\begin{tabular}{ll}
\hline Variable & $\mathbf{n}=\mathbf{3 0}$ \\
\hline Age (years) & $64.66 \pm 9.77$ \\
Height $(\mathrm{m})$ & $1.68 \pm 0.09$ \\
Weight $(\mathrm{kg})$ & $80.2 \pm 13.64$ \\
BMI $(\mathrm{kg} / \mathrm{m} 2)$ & $28.36 \pm 4.8$ \\
Sex ratio of male: female & $12: 18$ \\
Tender joint count (TJC) & $3.43 \pm 3.11$ \\
Swollen joint count (SJC) & $1.76 \pm 1.13$ \\
Disease duration, weeks & $15.53 \pm 21.87$ \\
Monoarticular disease & $10(33.3 \%)$ \\
Oligoarticular disease & $13(43.33 \%)$ \\
Polyarticular disease & $7(23.34 \%)$ \\
Acute arthritis & $23(76.66 \%)$ \\
Chronic arthritis & $7(23.34 \%)$ \\
\hline Values are express as mean \pm standard deviation or as number of \\
patients (\%); BMI - body mass index.
\end{tabular}


Table II. The mean US joint involvement per patient

\begin{tabular}{lll}
\hline Clinical forms & $\begin{array}{l}\text { Mean US joint involve- } \\
\text { ment }\end{array}$ & p-value \\
\hline Acute arthritis & $2.78 \pm 1.56$ & $\mathrm{p}=0.08$ \\
Chronic arthritis & $3.85 \pm 6.14$ & \\
Monoarticular disease & $3.12 \pm 2.07$ & $\mathrm{p}<0.05$ \\
Oligoarticular disease & $2.38 \pm 0.96$ & \\
Polyarticular disease & $4.14 \pm 2.34$ & \\
\hline
\end{tabular}

Values are express as mean \pm standard deviation

Table III. Joint distribution of CPP deposits evaluated by US

\begin{tabular}{llll}
\hline & \multicolumn{3}{l}{ Number of joints with CPPD } \\
\hline Joints & Total & Bilateral & Unilateral \\
\hline Acromio-clavicular & 7 & 3 & 1 \\
Gleno-humeral & 0 & 0 & 0 \\
Wrist & 17 & 7 & 3 \\
Hip & 3 & 1 & 1 \\
Knee & 58 & 28 & 2 \\
Ankle & 1 & 0 & 1 \\
Symphysis pubis & 2 & & \\
\hline
\end{tabular}

Table IV. The US patterns and calcification sites

\begin{tabular}{ll}
\hline Variable & N (\%) \\
\hline Pattern of calcification & patients \\
I - thin hyperechoic band & $10(33.33)$ \\
II - hyperechoic spots & $19(63.33)$ \\
III - hyperechoic nodular or oval deposits & $1(3.34)$ \\
Site of CPP deposits at all the joints & joints \\
Fibrocartilage & $62(70.45)$ \\
Hyaline cartilage & $22(25)$ \\
Fibrocartilage and & $4(4.55)$ \\
hyaline cartilage & joints \\
Site of CPP deposits at the knee joint & $19(32.76)$ \\
Medial meniscus & $14(24.13)$ \\
Lateral meniscus & $21(36.20)$ \\
Hyaline cartilage & $4(6.89)$ \\
Fibrocartilage and &
\end{tabular}

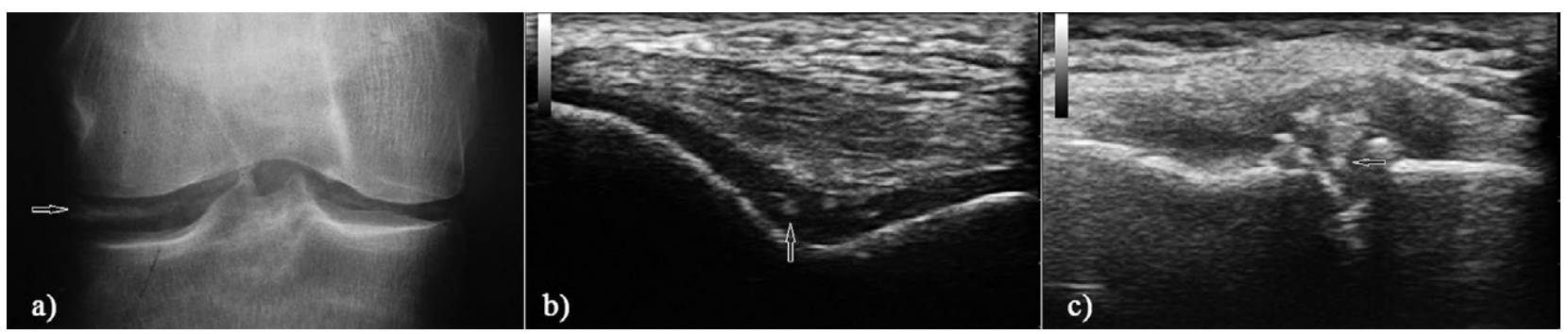

Fig 1. Radiographic and ultrasonography images of calcium pyrophosphate deposition: a) Conventional radiography showing meniscus calcifications (arrow); b) knee joint, transversal scans showing hyperechoic spots in the hyaline cartilage (arrow); c) knee joint, longitudinal scan of the medial compartment showing hyperechoic spots in the medial meniscus (arrow).

A total of 390 joints were scanned, in 30 patients. US calcifications were found in 88 joints $(22.56 \%)$.

\section{Distribution of affected joints}

The mean \pm SD number of joints with US evidence of CPPD per patient was $2.93 \pm 1.8$ (range 1-9) (Table II).

The most frequent CPP deposition was found in the knee followed by the wrist. Symphysis pubis involvement was found in two patients and all these two patients had a mean average of 5.5 joints affected by CPPD. Data regarding the distribution of the joints with CPPD evidence on US are summarized in Table III.

\section{US pattern and CPP deposits sites}

The second US pattern was the most common, followed by the first and the third pattern (Table IV). In addition, the second pattern was more frequent in patients with monoarticular or oligoarticular disease and in patients with acute arthritis (fig 2, Table IV).

Radiographic evidence of CPPD were found in 46 $(60 \%)$ knees (in 16 knees bilateral and in 14 unilateral).

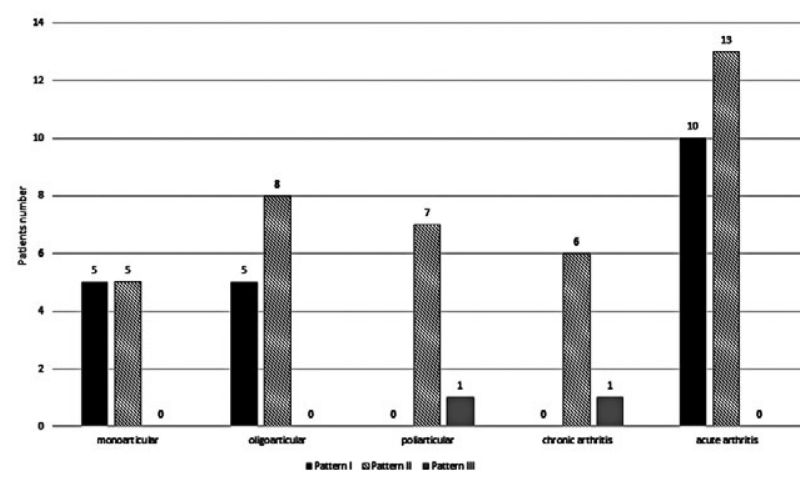

Fig 2. Ultrasonographic pattern of calcium pyrophosphate deposition distribution according to clinical findings (details inside the main text).

All patients had at least one knee joint with radiographic CPPD proof. Using radiography as the reference method, the sensitivity and specificity of US for diagnosis 
CPPD in knees was $79.31 \%, 95 \mathrm{CI}(66.65 \%-88.83 \%)$ and $14.29 \%$, 95CI(1.78\%-42.81\%), respectively.

\section{Discussions}

US is a useful, inexpensive, noninvasive, quick tool for detecting even small deposits of CPPD, with high specificity and sensitivity, and without irradiation risks. There are some studies, which evaluate CPPD by US, the most common at the knee joint, and only a few at multiple sites [8,9,13-17]. There is no agreement about which joints should be screened and no universal accepted ultrasound definition for CPPD. The most used definition for CPPD are those described by Frediani et al [7]. Recently, the Outcome Measures in Rheumatology (OMERACT) defined the US characteristics of CPPD in the fibrocartilage, hyaline cartilage, tendon and synovial fluid (reliable in the assessment of the knee fibrocartilage and hyaline cartilage) together with intra and interobserver reliability. The new definitions included the form, the shape, localization, and the behavior at dynamic scanning of the CPPD deposits [18].

To our knowledge, this is the first study which evaluates the 13 possible sites for CPPD using US.

The result regarding the clinical presentation, in terms of monoarthritis and oligoarthritis, also the female predominance were in agreement with other studies. The mean age in our study was lower compared with other studies $[17,19,20]$ but higher than Ellabban et al study [16]. Regarding the presentation form, as acute or chronic arthritis, our results were not in agreement with Filippou et al results. They found the chronic arthritis form as the most common [21]. In the same study, in which they assessed the extent and distribution of CPP deposits in 2-5 metacarpophalangeal joints, wrists, knees, Achilles tendon, and plantar fascia the authors found also the knee to be the most common joint affected by CPPD [21]. The same results were obtained when conventional radiography was used as a tool of investigation in the Abhishek et al study [22].

Filippou's et al study found the mean number of US affected joints (4.7) higher comparative with our study (2.93) probably because of different joints scanned and the multiple sites examined at the knee level. In addition, they did not find any correlation between clinical presentation and the number of involved sites by CPPD, contrary to our results. We observed that our patients who presented with an acute arthritis form or polyarticular disease had a mean US joint involvement significantly higher comparing with Filippou et al [21]. Another interesting finding was the frequency of the second ultrasound pattern in patients with monoarticular, oligoarticular disease and in pa- tients with acute arthritis. Ellaban et al conducted a study at multiple sites in which examined the shoulders, elbow, wrist, and knee joint by using US. Similar to our study, the knee was the most affected joint and the second US pattern (hyperechoic spots) was the most frequent [16] but no correlation between clinical data and US findings were found. The higher frequency of knee involvement in CPPD was found also in other studies [8,13].

We found no US study to examine the symphysis pubis in CPPD patients. In our study, the prevalence of US symphysis pubis involvement was $6.66 \%$. In a radiographic study of Abhishek et al the prevalence of CPPD in symphysis pubis was $3.6 \%$. In another two computed tomography studies, the prevalence of CPPD in symphysis pubis was $21.1 \%$ [23], and $12.5-27.5 \%$ [24]. Ramonda et al found in a radiographic study a prevalence for symphysis pubis involvement, of $33.1 \%$ [19].

In agreement with other studies, we found the fibrocartilage as the most frequent site of CPP deposits per total and also at the knee level [16,21]. With regard to medial versus lateral meniscus involvement by CPPD deposits, we found no significant differences between two sites, similar to Filippou et al [21].

Our results demonstrated high sensitivity and low specificity for US when radiography was considered as standard. These findings are not in agreement with other studies, which found a high specificity $[3,16]$.

Our study has some limitations. First, the study has a small number of patients. Also, we did not assess the intra and interobserver reliability for US and radiographic findings. The presence of CPP crystals was confirmed by microscopy only in the knee joints and we only assumed that in other joints the deposits are formed by CPP (this is, in fact, the main limitation of all other published studies). The lack of comparison of US with computer tomography and magnetic resonance imaging findings is another limitation of our study. Also, we did not evaluate by radiography all the joints scanned with US, especially due to the high dose of radiation need for all these sites.

\section{Conclusions}

US examination is a useful imaging technique to determine the distribution of CPP deposits. This is important especially because CPPD generally are present in more than one joint, not strictly related to clinical presentation. The knee joint and the wrist are the most common joints involved by CPPD, the hyperechoic spots is the most frequent US pattern and fibrocartilage is more frequently affected compared to the hyaline cartilage.

Conflict of interest: none. 


\section{References}

1. McCarty DJ. Calcium pyrophosphate dihydrate (CPPD) crystal deposition disease - nomenclature and diagnostic criteria. Ann Int Med 1977;87:240-242.

2. Zhang W, Doherty M, Bardin T, et al. European League Against Rheumatism recommendations for calcium pyrophosphate deposition. Part I: Terminology and diagnosis. Ann Rheum Dis 2011;70:563-570.

3. Foldes K. Knee chondrocalcinosis: an ultrasonographic study of the hyaline cartilage. Clin Imaging 2002;26:194-196.

4. Steinbach LS. Calcium pyrophosphate dihydrate and calcium hydroxyapatite crystal deposition diseases: imaging perspectives. Radiol Clin North Am 2004;42:185-205.

5. Swan A, Amer H, Dieppe P. The value of synovial fluid assays in the diagnosis of joint disease: a literature survey. Ann Rheum Dis 2002;61:493-498.

6. Genant HK. Roentgenographic aspects of calcium pyrophosphate dihydrate crystal deposition disease (pseudogout). Arthritis Rheum 1976;19 (Suppl 3):307-328.

7. Frediani B, Filippou G, Falsetti P, et al. Diagnosis of calcium pyrophosphate dihydrate crystal deposition disease: ultrasonographic criteria proposed. Ann Rheum Dis 2005;64:638-640.

8. Gutierrez M, Di Geso L, Salaffi F, et al. Ultrasound detection of cartilage calcification at knee level in calcium pyrophosphate deposition disease. Arthritis Care Res (Hoboken) 2014;66:69-73.

9. Filippou G, Frediani B, Gallo A, et al. A "new" technique for the diagnosis of chondrocalcinosis of the knee: sensitivity and specificity of high-frequency ultrasonography. Ann Rheum Dis 2007;66:1126-1128.

10. Backhaus M, Burmester GR, Gerber T, et al. Guidelines for musculoskeletal ultrasound in rheumatology. Ann Rheum Dis 2001;60:641-649.

11. Möller I, Janta I, Backhaus M, et al. The 2017 EULAR standardised procedures for ultrasound imaging in rheumatology. Ann Rheum Dis 2017;76:1974-1979.

12. Paparo F, Fabbro E, Ferrero G, et al. Imaging studies of crystalline arthritides. Reumatismo 2012;63:263-275.

13. Ruta S, Catay E, Marin J, Rosa J, García-Monaco R, Soriano E. Knee effusion: ultrasound as a useful tool for the detection of calcium pyrophosphate crystals. Clin Rheumatol 2016;35:1087-1091.
14. Grassi W, Meenagh G, Pascual E, Filippucci E. "Crystal clear" sonographic assessment of gout and calcium pyrophosphate deposition disease. Semin Arthritis Rheum 2006;36:197-202.

15. Gamon E, Combe B, Barnetche T, Mouterde G. Diagnostic value of ultrasound in calcium pyrophosphate deposition disease: a systematic review and meta-analysis. RMD Open 2015;1:e000118.

16. Ellabban AS, Kamel SR, Omar HA, El-Sherif AM, AbdulMagied RA. Ultrasonographic diagnosis of articular chondrocalcinosis. Rheumatol Int 2012;32:3863-3868.

17. Louthrenoo W, Sukitawut. Calcium pyrophosphate dihydrate crystal deposition: a clinical and laboratory analysis of 91 Thai patients. J Med Assoc Thai 1999;82:569-576.

18. Filippou G, Scirè CA, Damjanov N, et al. Definition and Reliability Assessment of Elementary Ultrasonographic Findings in Calcium Pyrophosphate Deposition Disease: A Study by the OMERACT Calcium Pyrophosphate Deposition Disease Ultrasound Subtask Force. J Rheumatol 2017;44:1744-1749.

19. Ramonda R, Musacchio E, Perissinotto E, et al. Prevalence of chondrocalcinosis in Italian subjects from northeastern Italy. The Pro. V. A. (PROgetto Veneto Anziani) study. Clin Exp Rheumatol 2009;27:981-984.

20. Rho YH, Zhu Y, Zhang Y, Reginato AM, Choi HK. Risk factors for pseudogout in the general population. Rheumatology 2012;51:2070-2074.

21. Filippou G, Filippucci E, Tardella M, et al. Extent and distribution of CPP deposits in patients affected by calcium pyrophosphate dihydrate deposition disease: an ultrasonographic study. Ann Rheum Dis 2013;72:2836-1839.

22. Abhishek A, Doherty S, Maciewicz R, Muir K, Zhang W, Doherty M. Evidence of a systemic predisposition to chondrocalcinosis and association between chondrocalcinosis and osteoarthritis at distant joints: A cross-sectional study. Arthritis Care Res (Hoboken) 2013;65:1052-1058.

23. Patel T, Ryan L, Dubois M, et al. The prevalence of chondrocalcinosis of the symphysis pubis on CT scan and correlation with calcium pyrophosphate dihydrate crystal deposition disease. Clin Rheumatol 2016;35:771-773.

24. Stensby JD, Lawrence DA, Patrie JT, Gaskin CM. Prevalence of asymptomatic chondrocalcinosis in the pelvis. Skeletal Radiol 2016;45:949-954. 